\title{
Osman II ou les infortunes de la Majesté : portrait d'un sultan « corrompu »
}

Robin Beuchat

\section{(2) OpenEdition}

1 Journals

Édition électronique

URL : http://journals.openedition.org/edl/919

DOI : $10.4000 /$ edl. 919

ISSN : 2296-5084

Éditeur

Université de Lausanne

\section{Édition imprimée}

Date de publication : 15 décembre 2015

ISBN : 978-2-940331-47-5

ISSN : 0014-2026

\section{Référence électronique}

Robin Beuchat, "Osman II ou les infortunes de la Majesté : portrait d'un sultan « corrompu » »,Études de lettres [En ligne], 3-4 | 2015, mis en ligne le 01 décembre 2018, consulté le 15 décembre 2020. URL: http://journals.openedition.org/edl/919; DOI : https://doi.org/10.4000/edl.919

\section{(C) Études de lettres}




\section{OSMAN II OU LES INFORTUNES DE LA MAJESTÉ: PORTRAIT D’UN SULTAN «CORROMPU»}

Le 22 mai 1622, l'Empire ottoman assiste à un événement sans précédent: l'exécution du sultan régnant, Osman II, par sa propre milice. Les chroniqueurs européens s'en emparent aussitôt pour proposer, au-delà du cas d'Osman, une réflexion sur la notion de «majesté» - cette «force vive, personnifiée dans le roi, et qui tient les sujets en son pouvoir», pour reprendre la définition de Ralph Giesey. Cet article cherche à ressaisir les enjeux de ce discours à la lumière de l'histoire anthropologique mais aussi de la poétique, car si Osman est une victime de l'Histoire, il devient rapidement, sous la plume de Tristan L'Hermite, un héros de tragédie.

Le 22 mai 1622, le sultan Osman II (1618-1622) est déposé par ses propres «esclaves", puis assassiné. L'événement est inouï, au sens littéral du terme: c'est la première fois, en plus de trois siècles d'existence, qu'un régicide est perpétré dans l'Empire ottoman. Ironie du sort, sa victime porte le même nom que le fondateur de la dynastie, Osman $1^{\text {er }}$ (v. 1280v. 1324).

Cette tragédie, dont l'écho parvient aussitôt en Europe, place au centre de la scène, sous une lumière crue, un corps, celui d'Osman: un corps jeune, en pleine santé, et au départ investi d'un pouvoir illimité; mais aussi - au fil que l'histoire avance - un corps fragile, et bientôt impuissant face au lacet des muets. Corps tout-puissant, corps misérable: on comprend que ce renversement de fortune spectaculaire, prenant à rebours l'image traditionnelle du sultan, ait suscité dans toute l'Europe chrétienne une réflexion sur le corps du sultan et, plus largement, sur l'exercice du pouvoir. Cette réflexion, bien sûr, n'a pas pris un tour théorique; elle s'est exprimée en acte, dans des récits appartenant à des genres variés. La mort d'Osman n'offre pas prise à une interrogation 
sur la corruption au sens biologique du terme, tel que par exemple le définit Furetière - "l'action par laquelle une chose se corrompt, se change, s'altere » ${ }^{1}$. Le cas de Soliman le Magnifique (1520-1566), décédé de mort naturelle et dissimulant, au soir de sa vie, les effets de l'«injure du tems ${ }^{2}$, s'y prête bien mieux. Mais, pour brutale et prématurée qu'elle soit, la mort d'Osman n'évacue pas pour autant le problème de la corruption; elle le transpose sur un autre plan, d'ordre symbolique. Elle nous offre en effet le spectacle d'un divorce entre les deux corps du sultan - entre, d'un côté, son corps biologique, individuel et caduque et, de l'autre, son corps mystique, désincarné et immortel ${ }^{3}$. En d'autres termes, c'est le corps d'un sultan "corrompu», indigne de sa fonction, qu'il s'agira d'autopsier dans les pages qui suivent.

Alors que le sultan ottoman jouit d'un prestige formidable aussi bien parmi son peuple qu'en Europe, le jeune Osman, qui accède au pouvoir à l'âge de quatorze ans, en 1618, épuise son crédit en quelques années. Il hypothèque sa Majesté et, partant, ouvre la voie à sa déposition et à son exécution. Désireux d'imposer une nouvelle forme à la dignité de sultan, il ne parvient au final qu'à accuser l'écart entre sa propre personne et le sultan idéal tel que l'imaginent ses sujets - et en premier lieu sa milice, ses «esclaves» comme on les appelait.

Si Osman contrevient très imprudemment aux usages dès son accession au trône, c'est, de l'avis général, la campagne de Pologne qui marque le début réel de cette crise ${ }^{4}$. Cette guerre qu'Osman avait entreprise "contre l'avis \& conseil de tous ses Visirs \& autres grands de son estat" s'était en effet soldée par "une grande desconfiture " ${ }^{5}$. Au-delà de l'échec militaire, elle avait contribué à instaurer un climat délétère entre le sultan et son armée. Osman, au début de la guerre, n’avait accordé qu'une maigre gratification à une minorité de ses soldats, ce

I. F. Furetière, Dictionnaire universel, art. «Corruption».

2. Le mot est d'Ogier Ghislain de Busbecq, ambassadeur de l'empereur Ferdinand $1^{\text {er }}$ de Habsbourg auprès de la Porte entre 1554 et 1562 . Voir Lettres du baron de Busbec, t. I, p. 192. Les Lettres turques paraissent en latin entre 1581 et 1589.

3. Notre réflexion sur les deux corps du sultan s'inscrit bien sûr dans la lignée des travaux d'E. Kantorowicz (The King's two Bodies). Pour une histoire anthropologique du sultanat ottoman, voir le beau livre de N. Vatin et G. Veinstein, Le sérail ébranlé.

4. On trouve un récit circonstancié de cette campagne dans le Septiesme tome $d u$ Mercure François, p. 762-779.

5. Ibid., p. 769 et 772 . 
qui, selon Tugi Efendi, avait entraîné "un grand desordre par après " ${ }^{6}$, l'ardeur de ses troupes s'en ressentant fortement. Le sultan, qui se sent trahi, aggrave ce climat en ne rémunérant à son retour de Pologne que les plus méritants de ses hommes et en traquant, de nuit, ceux qui s'encanaillent dans les tavernes de la capitale. Mal conseillé, il décide alors de partir en pèlerinage, ce qui soulève un concert de protestations au plus haut niveau de l'Etat. La rumeur se propage bientôt que, sous couvert d'un voyage de dévotion, il projette en réalité de lever une nouvelle milice et même de déplacer le siège de l'Empire en Asie. Les "esclaves de la Porte», qui occupent une place centrale dans l'Etat, s'en scandalisent et s'opposent au départ du sultan. Alors, sous le coup de la fureur, l'impensable se produit: Mustapha est hissé sur le trône à la place de son neveu, tandis qu'Osman est conduit à la prison des sept Tours, puis étranglé.

On voit par ce récit qu’Osman est largement responsable, sinon de sa propre mort, du moins des conditions qui l'ont rendue possible. C'est en effet lui qui, le premier, rompt le pacte tacite qui le lie à ses soldats, qui brise le cercle vertueux des devoirs réciproques. Dans cet esprit, le bayle de Venise Giorgio Giustinian évoque la longue série des écarts de conduite du sultan qui, cumulés, finissent par entraîner sa perte: le fait qu'il n'ait pas récompensé ses soldats, "comme ce fut toujours la coutume de ses aînés " ${ }^{7}$; qu'il se soit "écarté des coutumes de ses aînés " ${ }^{8}$ en épousant une fille libre, la fille du muphti; qu'il ait bafoué l'avis des docteurs de la loi, qui pourtant «furent toujours tenus en grande estime par ses aînés» ${ }^{9}$; ou encore qu'il ait décidé de partir en pèlerinage, une résolution "que les rois précédents n'avaient jamais prise " ${ }^{10}$. Ces transgressions répétées coûtent cher au sultan, elles le placent en

6. La Mort du Sultan Osman, ou le retablissement de Mustapha sur le Throsne, traduit d'un manuscrit Turc, de la Bibliotheque du Roy, par Antoine Galland, p. 21 sq. Le manuscrit original est attribué par N. Vatin et G. Veinstein à Tugi Efendi.

7. G. Giustinian, Relazione, p. 550.

8. Ibid., p. 551.

9. Ibid., p. 552.

Io. Ibid. G. Piterberg tire le même constat des chroniques ottomanes: "Sultan Osman was a nonconformist to an extent that, judging by his fate and the way he was treated and seen by contemporaries and by later generations, his society and polity could not tolerate» (An Ottoman Tragedy, p. 17). Aux raisons données par Giustinian, il ajoute le fait qu'Osman a cherché à raviver la tradition du sultan-guerrier, tombée en désuétude depuis longtemps, et qu'il a manqué d'un appui féminin. 
situation d'extrême précarité. Le fait même que ses actions soient tenues pour des infractions souligne son impuissance. Car le problème n'est pas tant qu'il s'écarte du modèle de gouvernance admis; c'est qu'il s'avère incapable d'imposer le nouveau modèle qu'il prétend incarner, celui d'un sultan véritablement absolu - au sens étymologique du terme: délié, parfaitement libre de ses actes.

Les effets de l'excentricité d'Osman ne se font pas attendre; ils se font sentir d'abord sur sa Majesté. Cette "force vive, personnifiée dans le roi, et qui tient les sujets en son pouvoir " ${ }^{11}$, telle que la définit Ralph E. Giesey, est un concept essentiel du pouvoir monarchique. Elle donne en effet à voir l'invisible, l'intime liaison entre les deux corps du roi: entre, d'une part, son corps mystique, supra-individuel et éternel, du moins en droit, et, d'autre part, son corps individuel, biologique et caduque (celui de l'individu qui, pour un temps seulement, est dépositaire de la dignité monarchique). C'est, pour cette raison même, le mode d'exercice du pouvoir par excellence. Les souverains ottomans, qui, se soustrayant la plupart du temps aux regards, n'apparaissaient que sur le mode de l'exhibition, de l'éclat, en étaient au reste parfaitement conscients. Nombreux sont les voyageurs qui ont décrit les apparitions théâtralisées du sultan. Parmi eux, le P. Pacifique de Provins, un capucin en mission en Orient, évoque la sortie "en triomphe» d'Osman au lendemain de ses noces controversées avec la fille du muphti:

[...] quelques jours apres nous ayant esté dit que le grand Seigneur sortiroit en triomphe le lendemain de son mariage avec sa seconde femme qui est la fille du Mouphety, nous le fusmes voir passer où il alloit du vieil Serrail où sont les femmes des deffunts grands Seigneurs, faire sa priere à la Mosquée de Sultan, il marchoit en cét ordre ${ }^{12}$.

Et Pacifique de décrire dans l'ordre de son déroulement le spectacle royal auquel il eut le privilège d'assister (le lecteur n'a malheureusement pas la même chance, puisque le passage est escamoté à cause d'une erreur d'impression).

Ce défilé, toutefois, ne doit pas faire illusion. Loin de faire honneur à la Majesté ottomane, Osman, de l'avis général, l'a au contraire dégradée,

II. R. E. Giesey, Cérémonial et puissance souveraine, p. 74.

I2. Pacifique de Provins, Relation du voyage de Perse faict par le R.P. Pacifique de Provins, predicateur capucin, p. 34. 
avilie. D'apparence austère, ce qui lui valut d'être traité de "vagabond" par le chroniqueur Mustafa Naima ${ }^{13}$, il n'a cessé d'agir de manière à dilapider son capital de Majesté. Ses rondes punitives sont ainsi unanimement décrites comme des actions indignes de son statut. A ce propos, l'ambassadeur d'Angleterre Thomas Roe note qu'Osman ne serait pas tombé si bas "s'il n'avait d'abord perdu l'estime (awe) et le respect (reverence) dus à la Majesté (Majestie)", s'il ne s'était pas rabaissé au statut d'homme "commun" (common) et «vulgaire» (cheape) ${ }^{14}$. Roe insiste ainsi sur l'écart qu'Osman creuse entre celui qu'on lui demande d'être (le sultan) et le personnage méprisable qu'il devient sans s'en rendre compte parce qu'il ne parvient pas à distinguer ce qui est noble et ce qui ne l'est pas, ce qui est important et ce qui est insignifiant (en l'occurrence le fait que les janissaires dérogent à la loi islamique qui interdit de boire de l'alcool). Giustinian, en moins de mots, fait le même constat:

[...] ces opérations, et d'autres du même type, étaient vraiment contraires à la dignité et à la majesté d'un si grand prince (indegne del decoro et maestà di tanto principe) $[\ldots]^{15}$.

Et Tugi Efendi joint sa voix à ce concert de reproches en notant qu'Osman aurait fait «des actions [...] peu dignes d'un Sultan» en voulant «empecher que les Spahis \& Janissaires ne pussent s'enyvrer $[\ldots] »{ }^{16}$.

Osman, longtemps obstiné et sûr de son bon droit, ne mesurera les conséquences de ses actes qu'en plein tumulte, lorsqu'il essaie d'imposer sa présence aux rebelles comme une force agissante. Vittorio Siri relate cette désillusion, cet excès de confiance qui butte sur le corps mort de son grand vizir, de même que la phrase achoppe sur le connecteur «mais»:

13. Voir G. Piterberg, An Ottoman Tragedy, p. 20.

I4. Th. Roe, "The Death of Sultan Osman, and the setting up of Mustafa his Uncle, according to the Relation Presented to His Majestie», p. 354 sq. On lit également cette relation, d'abord destinée au secrétaire d'Etat George Calvert, dans la correspondance stambouliote de Roe, The Negotiations of Sir Thomas Roe in his Embassy to the Ottoman Porte, from the Year 1621 to 1628 inclusive, p. 45-52.

I5. G. Giustinian, Relazione, p. 551.

I6. T. Effendi, La Mort du Sultan Osman, p. 19. 
Mais sultan Osman, [...] se persuadant qu'il pouvait apaiser le tumulte en opposant aux rebelles sa seule présence (la sola sua presenza), sortit du sérail accompagné de ses Vizirs et de tous les insignes ordinaires de la Majesté Ottomane (da tutte l'Insegne ordinarie della Maestà Ottomana) pour aller à leur rencontre; mais en s'approchant de ces furieux il vit à ses pieds, mort sous de multiples coups, le grand Vizir $[\ldots]^{17}$.

Le comte de Césy, ambassadeur de France auprès de la Porte, s'étonne quant à lui de voir ce prince «sy indignement traitté par les rues où il avoit passé depuis peu en sy grande pompe $[. ..]{ }^{18}$, brossant un contraste d'autant plus frappant qu'on passe d'un extrême à l'autre - de la vénération au mépris - en un instant à peine. Dans le même esprit, mais sans passion, Michel Baudier extrapole de ces événements tragiques une réflexion sur l'avilissement de "la Monarchie des Sultans des Turcs»:

Estrange mespris de la Majesté des Sultans des Turcs, elle a par l'espace de trois cens vingt-deux ans paru dans l'Orient avec tant d'esclat, \& receu tant de reverence, que celuy-là estoit arrivé dans le Serrail au comble d'une grande faveur, quand il avoit le tiltre de Musaip, c'est à dire, qui peut parler au Prince. Cette Majesté est maintenant avilie, mesprisee, $\&$ a pour compagnie un bourreau dans ce chariot [qui conduit Osman vers la prison des sept Tours] ${ }^{19}$.

Tugi-Galland va plus loin. Abordant lui aussi la question de la présence d'Osman et de ses effets politiques, il constate que celui-ci, au plus fort de la crise, n'est pas même "en estat de se faire voir» ${ }^{20}$. Autrement dit, il s'en prend à sa santé. Non content de le déposséder de son charisme royal, il le prive également de ses ressources physiques, ce qui est d'autant plus discriminant que, dans la société ottomane, il fallait être en mesure

17. V. Siri, Il Mercurio overo historia de' correnti tempi, tome I, p. 170-173.

I8. Advis de Constantinople du xxix May 1622, BnF, ms. fr. 3794, pièce nº 9 , ff. 16 $\mathrm{r}^{\circ}-17 \mathrm{v}^{\circ}$; f. $17 \mathrm{v}^{\circ}$. Cette lettre adressée à Louis XIII (demeurée inédite) est reprise en partie dans le Huictiesme tome du Mercure François (Paris, Jean Richer, 1623, p. $357-$ 374), qui propose un montage à partir de plusieurs sources (outre l'Advis de Césy, la Lettre du P. Pacifique et la Tragoedic Constantinopolitane Descriptio de Michael Caspar Lundorp dit Lundorpius, parue dans le livre III de son Belli sexennalis civilis Germanici, Francofurti, J. T. Schonwetterus, 1623, p. 15-22).

19. M. Baudier, Inventaire de l'Histoire generalle des Turcz, p. 883.

20. T. Effendi, La Mort du Sultan Osman, p. 106. 
de prouver sa bonne santé pour prétendre régner ${ }^{21}$. Souverain indigne et impotent, dépourvu aussi bien de Majesté que de vitalité, Osman est doublement et définitivement disqualifié:

[...] comme on vit que la sedition $\&$ le bruit augmentoient toûjours, plusieurs Efendis jugerent à propos de leur [aux rebelles] faire voir Osman, pour tenter si sa presence ne les appaiseroit pas. Ils le firent monter sur un Cheval du Muphti Ashad Efendi; mais il estoit si fort allarmé \& si foible, qu'il ne pût se tenir à cheval, \& fût obligé de mettre pied à terre $\&$ de rentrer dans la Chambre des Requestes, le peu de resolution qu'il avoit ne luy permettant pas d'aller plus loing ${ }^{22}$.

Le sultan échappe toutefois, dans un contexte générique certes particulier, à ce processus d'avilissement: dans la tragédie de Tristan L'Hermite, Osman (probablement représentée en 1646 et publiée à titre posthume en 1656) ${ }^{23}$. Prenant à rebours ses sources historiques, Tristan décide en effet de rendre sa Majesté au sultan, de métamorphoser le "pauvre petit Prince» de Pacifique en héros cornélien. On le voit tout particulièrement dans le coup d'éclat qu'un dénommé Musulman raconte à la fille du Muphti, qui se caractérise par des sentiments ambivalents à l'égard du sultan (elle l'aime, mais veut se venger de lui). Osman, assailli par les rebelles, semble d'abord en mauvaise posture. En un moment, "vingt mille hommes armés» se sont réunis, "poussant mille cris qui montaient jusqu’aux nues"; et le récit de se poursuivre:

Ils en gagnaient déjà toutes les avenues,

2I. Voir N. Vatin et G. Veinstein, Le sérail ébranlé, p. 30-36.

22. T. Effendi, La Mort du Sultan Osman, p. 105 sq.

23. Tristan L'Hermite, Osman [1656], in Euvres complètes, t. IV, p. 461-542. Sur les sources d'Osman, voir N.-M. Bernardin, Un précurseur de Racine, p. 469-472; H. C. Lancaster, A History of French Dramatic Literature in the Seventeenth Century, vol. II, p. 569; C. D. Rouillard, The Turk in French History Thought and Literature (1520-1660), p. 496. On consultera également avec profit l'appareil de notes de l'édition procurée par N. Mallet qui, sur la base des travaux ci-dessus, apporte des précisions sur les emprunts de Tristan. En complétant ces études par nos propres recherches et sans entrer ici dans les détails, nous pouvons établir que Tristan utilise et détourne le Mercurio de Siri, la Lettre de Pacifique (qu'il a lue soit dans sa version originale, soit dans les Histoires tragiques de Malingre qui en donnent une copie conforme), de même que le montage paru dans le Huictiesme tome du Mercure François. Ses informations sur la campagne de Pologne proviennent quant à elles du Septiesme tome du Mercure François. 
Lorsque, pour effrayer les chefs de ce parti,

Les portes s'entrouvrant, Osman en est sorti,

Et s'est conduit au pas vers cette multitude,

Qui ne l'a vu venir qu'avec inquiétude ${ }^{24}$.

Ce passage, qui nous fait basculer dans la fiction, marque l'entrée en scène d'Osman, une entrée théâtralisée par la rétention, jusqu'au dernier moment, du sujet grammatical de la phrase - Osman. Construit comme un objet de regard, le sultan, métamorphosé par Tristan, parvient ici à imposer la force tranquille de sa présence:

Etant seul à cheval, sa personne admirable

Aux yeux de tous était plus vénérable ${ }^{25}$.

Couvert d'insultes dans les chroniques, Osman apparaît ici comme l'objet d'une vénération unanime ("aux yeux de tous»), et Tristan rehausse son "auguste aspect» en décrivant la splendeur, tout orientale, de son costume (la "couleur locale" était ainsi mise au service de l'éclat du prince). En un mot, il donne à voir une sortie d'Osman «en triomphe", là où le sultan historique était voué à l'opprobre publique. Et lorsqu'il lui cède la parole, ce n'est pas pour l'écouter se lamenter sur son sort; c'est pour lui donner l'occasion de revendiquer son identité héroïque, fondée sur une parfaite correspondance entre l'être, le paraître et le faire:

Ne suis-je pas Osman, de l'ottomane race?

Qui fais trembler la terre à mon auguste aspect,

Et qui sers le Prophète avec humble respect?

A t'on pu remarquer quelque sujet de blâme

Entre mes actions, même au fond de mon âme,

Pour vouloir abaisser à de serviles lois

Celui qui sous ses pieds tient les têtes des rois? ${ }^{26}$

Nulle dissimulation chez Osman: ses actions traduisent le «fond de [s]on âme» et honorent à la fois son nom et son sang. Cet autoportrait, on le voit, renverse plaisamment l'image d'un Osman indigne et commettant

24. Ibid., III, 2, v. 724-728.

25. Ibid., v. 731-732.

26. Ibid., v. $752-758$. 
des "actions inconvenantes" (unseemely offices) ${ }^{27}$, tel que l'a décrit Thomas Roe. Le sultan est aussi, chez Tristan, un maître orateur. Il parvient en effet à retourner contre ses propres détracteurs l'acte d'accusation dont il est d'abord l'objet, à créer une scène judiciaire fictive de manière à en monopoliser tous les rôles. La forme du monologue, ici, trahit et impose un absolutisme sans bornes et triomphant.

Au moyen de ce coup de théâtre - qui est aussi un coup d'Etat au sens où l'entendait Naudé: un acte extraordinaire entrepris par le souverain légitime ${ }^{28}$-, Tristan réhabilite la figure du prince en Majesté. Car son héros, ici, est plus le prince tragique idéal qu'Osman (en tant que figure historique) ou que le sultan ottoman (en tant que type de souverain). Comme pour se laver de tout soupçon, il prend d'ailleurs soin, à la fin de sa tragédie, d'annoncer la chute de l'Empire ottoman. Ce qu'il promeut, c'est une figure monarchique tout idéale, et même apolitique: Osman, chez lui, devient le prétexte d'une exaltation poétique du monarque absolu écrasé par son destin tragique.

Pour revenir aux textes plus fidèles à la réalité historique (qui sont aussi les plus nombreux), on note que la perte de Majesté d'Osman met en avant, comme par contraste, son corps biologique. Ainsi, chez Pacifique, la nudité d'Osman, privé des symboles du pouvoir - en l'occurrence de son turban -, devient la figure, éminemment pathétique, de son dénuement:

Si jamais il s'est veu au monde un object excitant à la compassion c'estoit de voir ce pauvre petit Prince monté sur ce cheval, avec sa cuirasse blanche, on luy avoit osté son Turban Royal, \& estoit tout teste nuë, la teste raze comme sont les Turcs, $\&$ avoit seulement une meschante petite calotte sur la teste, les larmes grosses comme des perles qui luy couloient le long des joües [... ${ }^{29}$.

27. S. Purchas, Hakluytus Posthumus, p. 354.

28. Naudé définit plus précisément les coups d'Etat comme des «actions hardies et extraordinaires que les princes sont contraints d'exécuter aux affaires difficiles et comme désespérées, contre le droit commun, sans garder même aucun ordre ni forme de justice, hasardant l'intérêt du particulier, pour le bien du public.» (Considérations politiques sur les coups d'Etat, p. 101).

29. Pacifique de Provins, Lettre du Pere Pacifique de Provin [sic], predicateur Capucin, estant de present à Constantinople, envoyee au R.P. Joseph le Clerc, Predicateur du mesme Ordre, \& Deffiniteur de leur Province de Tours, p. 18 sq. Le père Pacifique, qui voyage en Orient à la fois en missionnaire et en pèlerin, dit tenir ses informations de témoins 
Tout se passe comme si la déchéance d'Osman révélait, par contraste, ce qu'on peut appeler sa "corporéité» - sa matérialité précaire. Privé de son turban royal, Osman apparaît "tout teste nuë». Autrement dit, à la plénitude (symbolique) du pouvoir succède un corps nu, mis à nu - dans les deux sens du terme. L'insistance sur les larmes du sultan, "grosses comme des perles", revêt la même fonction; mais elle annonce de plus l'épanchement d'un autre liquide corporel, celui-là vital: le sang. Pacifique ne manque pas en effet d'évoquer la "grande quantité de sang ${ }^{30}$ qui s'écoule hors de la dépouille d'Osman mort, une mention d'autant plus significative qu'elle fait écho, quelques lignes plus haut, au «sang Royal», mystique, du sultan:

He! n'y a-il personne icy qui me vueille prester un poignard, pour me donner le moyen de venger ma mort, \& me deffendre contre mes bourreaux, mais en vain faisoit il [Osman] ces demandes; c'estoit la jeunesse \& le sang Royal, qui boüillant dans ses veines, ne luy pouvoient permettre d'envisager la mort ${ }^{31}$.

Les deux corps du roi se trouvent ainsi associés à travers le (double) sang d'Osman. Cette scène est d'autant plus frappante qu'elle transgresse un tabou. Il était en effet formellement interdit, en Turquie, de répandre le sang des fils d'Osman, qui était le seul fondement de l'Etat. L'association entre le sang mystique de la "race" ottomane et le sang biologique d'Osman se répandant sur le sol est donc extrêmement audacieuse. A la lumière des croyances politiques ottomanes, elle prend même une dimension sacrilège.

Au chapitre des sacrilèges, Pacifique en observe un second: le sultan, sitôt mort, aurait été mutilé (un détail dont le capucin, parmi les sources chrétiennes, est le seul à faire mention ${ }^{32}$ ):

directs. Rédigée à Péra, en face de Constantinople de l'autre côté de la Corne d'Or, et datée du 30 mai 1622, sa "Lettre", on l'a dit, est en partie reprise dans le Mercure François. Dans le même esprit que Pacifique, Roe relève qu'un spahi insolent aurait échangé son turban avec celui d'Osman (Hakluytus Posthumus, p. 348).

30. Pacifique de Provins, Lettre du Pere Pacifique, p. 23.

31. Ibid., p. 22.

32. Cela ne signifie pas qu'il soit forcément fictif. D’après G. Piterberg (An Ottoman Tragedy, p. 28 et 209, n. 44), Ibrahim Peçevi (1574-1650) mentionne un épisode similaire, à la différence près que l'oreille d'Osman, une fois coupée, aurait été apportée non pas à Mustapha, mais à la sultane mère. 
La cruauté et l'ignominie ne sont point encore cessées, ainçois elles vont commencer sur le corps du deffunct. Car si tost qu'il fut mort, le grand Vezier present, luy couppa une aureille qu'il mist dans son mouchoir, \& l'apporta à Sultan Mustapha pour l'asseurer que son Nepveu Osman estoit mort ${ }^{33}$.

Cette mutilation a beau être purement utilitaire (et donc ne pas participer d'un rituel d'humiliation), on ne peut s'empêcher de penser que, dans l'esprit de Pacifique, elle revêt une dimension hautement symbolique. Elle lui inspire, en tous les cas, un cri d'horreur: "O cruauté plus que barbaresque! O spectacle cruel!» ${ }^{34}$ Car c'est une chose de supplicier un corps vivant; c'en est une autre de mutiler une dépouille mortuaire. En passant d'un crime à l'autre, on franchit un degré dans l'horreur. Comme l'écrit Montaigne, "le peuple s'effraie des rigueurs qui s'exercent sur les corps morts» ${ }^{35}$. Pacifique, en qualifiant Osman de "petit prince» ${ }^{36}$, donne de plus à son assassinat l'allure d'un infanticide; et il le place d'entrée de jeu sous le signe d'une condamnation biblique, tirée du livre des Rois: «La paix peut-elle estre à Zambri, qui mit à mort son maistre? " ${ }^{37}$ D'une certaine manière, et aussi surprenant que cela paraisse, Osman est même assimilé au Christ. Un faisceau d'indices en tous les cas le suggère. On l'accuse d'abord d'être infidèle ("Jaour»), une accusation qui, pour infondée qu'elle soit, n'en tire pas moins le sultan du côté des chrétiens:

[...] ce qui donnoit de la surcharge à sa douleur estoit les paroles $\&$ actions impudentes que quelques soldats enragez luy disoient $\&$ faisoient par despit, l'un grinçoit les dents l'appelant Jaour, l'autre crachoit contre terre $\&$ frappoit du pied, $\&$ un entre les autres luy monstrant

33. Pacifique de Provins, Lettre du Pere Pacifique, p. 23.

34. Ibid.

35. M. de Montaigne, Journal de voyage, p. 198. Voir aussi dans les Essais, «De la cruauté», II, 11, p. 432 a.

36. On trouve ainsi «pauvre jeune Prince» (p. 12), "petit Prince Sultan Osman» (p.15), "jeune Prince Osman» (p. 16), "pauvre petit Prince» (pp. 18 et 20), "petit Prince» (pp. 19 et 25), «jeune Prince» (p. 20 et 25).

37. Epigraphe figurant en page de titre. Pacifique indique comme référence 4. Reg 9; on trouve en réalité l'histoire de Zambri, ou Zimri, dans 1 Rois 16.8-22. 
une chorde luy dist. O larron! tu meriterois d'estre estranglé avec cette chorde, comme un larron qui a voulu desrober nos thresors $[\ldots]^{38}$.

Le fait que le jeune prince lance des soupirs vers le Ciel, qu'il prie Dieu avec ferveur soulignent de plus sa religiosité, une religiosité sinon explicitement chrétienne, du moins dénuée de toute référence à l'islam: il n'est ainsi question que du «cœur affligé» d'Osman, que du «Ciel». D'autres aspects encore rapprochent le pauvre Osman de l'«innocent Jesus» dont Pacifique, qui a fait le pèlerinage de Terre sainte, relate la Passion dans son récit de voyage ${ }^{39}$. Comme le Christ, que ses bourreaux «despoüillerent tout nud» 40 , Osman se retrouve "tout teste nuë». Comme le Christ (victime de "mille opprobres») ${ }^{41}$, on le couvre d'insultes, et celle de «larron» ne manque pas de faire écho à l'épisode de la Crucifixion. Enfin, leurs morts respectives, décrites dans les mêmes termes - comme de lentes agonies -, se répondent en miroir. Lisons d'abord le récit de la mort d'Osman :

Le pauvre petit Prince se sentant ainsi serré [par le lacet des muets], \& aux dernieres agonies, se demena si courageusement des pieds $\&$ des mains, qu'ils avoient peine à l'estrangler, ce que voyant un de ces bourreaux, il luy lascha deux coups d'une petite hache, l'un sur l'espaule, l'autre sur le col, seulement pour l'estourdir, \& luy debilita si bien les forces, que ne se pouvant plus revancher ils l'estranglerent à leur aise, voila donc nostre petit Prince mort, \& son corps tombé par terre, rendant une grande quantité de sang par le nez \& par la bouche ${ }^{42}$.

Voici à présent celui de l'«agonie» du Christ:

Durant que nostre Seigneur Jesus combattoit contre la mort dedans son agonie, \& la perte de son sang, eslevé qu'il estoit en haut, voilà le Ciel qui se couvre de tenebres, la terre tremble, les rochers se fendent, $\&$ les corps morts qui sortent de leurs sepulchres, ouvertes par le tremblement de terre qui se fit lors, $\&$ entre les rochers qui se fendirent, le

38. Pacifique de Provins, Lettre du Pere Pacifique, p. 19.

39. Id., Relation du voyage de Perse, p. 187-219 («La Passion de Jesus de Nazaret, Fils de Dieu, \& de Marie»). La référence à «l'innocent Jesus» est à la page 202.

40. Ibid.

4I. Ibid.

42. Pacifique de Provins, Lettre du Pere Pacifique, p. 22 sq. 
mesme mont de Calvaire fut le plus notable, qui se depeça, $\&$ fendit Jesus $\&$ le mauvais larron $[\ldots]^{43}$.

Le combat prolongé contre la mort (ce que Jean Rousset appelait «la mort en mouvement»), la mention du sang, l'empathie pour la victime (soulignée dans les deux cas par l'usage du possessif affectif «nostre»): tout rapproche ici ces deux figures qu'a priori pourtant tout sépare. Tout se passe comme si Pacifique, probablement sensibilisé à la souffrance sainte par sa culture franciscaine, ne pouvait pas s'empêcher de christianiser l'innocent martyrisé qu'est Osman. Ce phénomène n'est pas sans rappeler ce que François Hartog a appelé «la règle du tiers exclu», selon laquelle «il semble [...] que, dans son mouvement pour traduire l'autre, le récit soit finalement incapable de prendre en charge plus de deux termes à la fois. ${ }^{44}$ En l'occurrence, l'absence de chrétiens sur la scène tragique semble rendre possible la christianisation de la victime turque. Pacifique, prisonnier d'un schéma manichéen - celui de la martyrologie et de la tragédie de collège d'inspiration sénéquienne -, semble incapable d'imaginer une victime autre que chrétienne, fût-elle le chef de file des infidèles. C'est à n'en pas douter l'incapacité de dépasser cette alternative qui vaut à Osman de devenir, sous la plume du capucin, une sorte de figure monstrueuse: un sultan-Christ.

Au-delà de l'acte de mutilation dont elle est l'objet chez Pacifique, la dépouille d'Osman joue un rôle fondamental. Par sa présence muette, qui rappelle que l'impensable s'est produit, elle pose la question de l'après violence. Chez Césy, les soins dont elle est l'objet, et le regret qu'elle alimente, en font un lieu d'apaisement. On a bafoué le vivant, on vénérera le mort:

Le sabmedy $\mathrm{xxi}^{\mathrm{e}}$ on apporta son corps [celui d'Osman] au grand Sarrail pour le laver et faire quelques autres ceremonies qu'observent les Mahometans et sur le midy les Vizirs assisterent à son enterrement sans aucune pompe mais avec beaucoup de larmes tant de ceux de la loy que du peuple tesmoignant tous un extresme regret de ce que la milice estoit cause de cette mort ${ }^{45}$.

43. Pacifique de Provins, Relation du voyage de Perse, p. 203.

44. F. Hartog, Le Miroir d'Hérodote, p. 392.

45. Advis de Constantinople du xxix May 1622, f. $17 \mathrm{v}^{\circ}$. 
Le respect des coutumes funéraires signale un retour à l'ordre: faire acte d'allégeance à la tradition est une manière de refermer cette parenthèse scandaleuse qu'est le régicide d'Osman, de panser une plaie encore vive. Comme le constate non sans ironie Thomas Roe, il en va de même des pleurs versées sur le cadavre du sultan:

[...] après s'être emportés au-delà des bornes de la raison, voilà qu'ils pleurent leur Roi défunt, conscients d'avoir souillé leur honneur en trahissant pour la première fois l'un de leurs Empereurs [... ${ }^{46}$.

"Ils pleurent leur Roi défunt", lit-on; mais ces larmes sont-elles unanimes? Les tenants du parti légitime - Césy et Pacifique - refusent de le croire. Césy trace ainsi une fracture claire entre, d'un côté, "ceux de la loy» et "le peuple» et, de l'autre, «la milice», qu'il tient pour responsable in corpore du meurtre d'Osman. Bref, il ne pardonne pas aux régicides, pas plus que Pacifique, qui accuse de surcroît la milice de censurer l'émotion publique:

Cet enterrement fut si triste, \& avecque si peu de pompe ny compagnie, que personne n'osoit assister; de peur d'estre soupçonné avoir esté de son party, seulement y avoit-il des femmes qui par leurs larmes \& paroles, appeloient tout haut le Ciel à tesmoin de cet outrage commis en la personne de ce jeune Prince ${ }^{47}$.

A l'inverse, Tugi prend fermement la défense des «esclaves de la Porte». Cela n'a rien pour surprendre: ce chrétien islamisé, ancien janissaire et ancien garde du corps du sultan ${ }^{48}$, appartenait en effet au "parti» des rebelles. Animé donc d'un esprit d'amnistie, il écrit que «[q]uand on eût publié qu'Osman estoit mort, toute la Ville fût dans une extreme consternation ». Et de poursuivre en insistant sur le sentiment de culpabilité (non fondé) des rebelles, c’est-à-dire sur leur humanité: «Les plus mutins, écrit-il, se repentirent de leur sedition \& s'imputerent une mort

46. S. Purchas, Hakluytus Posthumus, p. 349.

47. Pacifique de Provins, Lettre du Pere Pacifique, p. 25.

48. Pour une notice biographique de Tugi, voir G. Piterberg, An Ottoman Tragedy, p. 45. 
si injuste $[\ldots]{ }^{49}$. Son but, ici comme ailleurs, est d'innocenter les troupes de la capitale ${ }^{50}$.

Tristan, sur le même thème - celui de la dépouille d'Osman -, propose une variation tout à fait originale. Comme Pacifique, il mutile son héros; mais, d'une part, il aggrave la portée de cet acte - il fait décapiter Osman - et, de l'autre, il lui confère un tout autre sens. Le corps mutilé d'Osman donnait à Pacifique l'occasion d'exprimer sa sainte indignation et de rappeler, en filigrane, le respect dû aux morts. Tristan, lui, se sert de la tête d'Osman pour affirmer un principe de "politique poétique" ${ }^{51}:$ l'incorruptibilité de la Majesté royale. Même brandie au bout d'une pique, la «tête héroïque» d'Osman continue en effet de rayonner; le rebelle Mamud ne peut que le constater:

Ce chef si glorieux, cette tête hérö̈que

Est portée au sérail sur le fer d'une pique.

On dirait qu'elle jette un regard menaçant,

Que d'un feu de vengeance elle éclaire en passant

Et l'un de nos dervis remarque en ce visage

De nos prochains malheurs un assuré présage ${ }^{52}$.

Le discours de Mamud est certes modalisé ("on dirait»), mais peu importe qu'on se situe dans le domaine des apparences, puisque c'est précisément celui du pouvoir en acte. Osman réussit un véritable tour de force: même mort, il parvient à renverser le rapport de forces en sa faveur. On voulait l'humilier, exhiber sa tête en guise de trophée; voilà que s'affiche sur son visage le signe de la vengeance qui s'abattra sur ses ennemis. C'est une victoire certes paradoxale, mais c'est une victoire incontestable, dont on peut tirer la leçon suivante: le roi, dans le monde de Tristan, ne meurt jamais. Tout en s'apparentant au célèbre adage illustrant la théorie des deux corps du roi («le roi est mort, vive le roi!»), cette maxime s'en distingue sur un point capital: en ce qu'elle suppose, non pas l'immortalité du corps mystique du roi, mais l'incorruptibilité de la Majesté personnelle du roi - en l'occurrence d'Osman. C'est là la

49. T. Effendi, La Mort du Sultan Osman, p. 175.

50. C'est ce que G. Piterberg appelle la «signification latente» de son récit $(A n$ Ottoman Tragedy, p. 70-90).

5I. J'emprunte cette notion à H. Merlin-Kajman à propos de Corneille (L'absolutisme dans les lettres et la théorie des deux corps, p. 62).

52. Tristan L'Hermite, Osman, V, 3, v. 1572-1575. 
profonde originalité de la "politique poétique» de Tristan ${ }^{53}$, que la fille du Mufti, interrompant Mamud, énonce à sa manière:

C'est assez, c'est assez, de grâce, arrête toi!

On n'a rien fait encore, on ne peut rien sans moi.

Quoi que fidèlement ta bouche me raconte,

L’impérieux Osman vit encore à ma honte ${ }^{54}$.

"L’impérieux Osman vit encore»: le héros tristanien ne meurt jamais; il continue à vivre et à resplendir de son éclat, pour l'éternité, dans le présent de la mémoire - de la mémoire visuelle; citons encore la fille du Mufti:

Je le vois, ce grand prince au point d'un partement,

Qui fait connaître aux siens son mécontentement;

Je l'aperçois qui m'aime et qui me persécute,

Qui brave les malheurs et qui leur sert de butte.

Je vois son port auguste et plein de Majesté,

Qui relève l'éclat d'une mâle beauté,

Et vois même briller parmi l'air qu'il respire,

La grandeur ottomane et celle de l'Empire ${ }^{55}$.

On le voit, le régicide d'Osman donne lieu à une réflexion féconde sur le pouvoir ottoman. Il permet de s'interroger sur ses fondements, sa nature, son mode d'exercice, mais également sur ses limites - car, en dépit des lieux communs, le souverain ottoman n'est pas absolu. Il permet également d'éprouver la fragilité, la "corporéité» du sultan, dès lors que celui-ci n'est plus protégé par le mystère de sa fonction - un mystère lié à la nature de sa fonction, mais savamment cultivé par la tradition. Au-delà du cas ottoman, il apparaît enfin comme le support idéal d'une méditation sur le corps souffrant: Pacifique peut ainsi associer Osman au Christ en Croix, et Tristan le transformer en héros tragique.

53. Il annonce en ce sens le moi royal de Louis XIV tel que le décrit R. Giesey, Cérémonial et puissance souveraine, p. 85: «Le cas de Louis XIV est particulièrement clair: il a abandonné son moi privé au profit d'une véritable incarnation du pouvoir souverain. Vu autrement, ce processus aboutit à une personnalisation de cette incarnation, jadis entité fictive, ou mystique, idéale et invariante, à laquelle les rois successifs avaient à s'ajuster".

54. Tristan L'Hermite, Osman, V, 4, v. 1578-1581.

55. Ibid., v. 1590-1597. 
Les récits chrétiens de la mort d'Osman, "baroques" au sens où ils abordent à leur manière tous les thèmes chers à Jean Rousset - «le changement, l'inconstance, le trompe-l'oil et la parure, le spectacle funèbre, la vie fugitive et le monde en instabilité " ${ }^{56}$ - sont ainsi hantés par l'idée de la corruption, par l'intime conscience de la précarité du pouvoir et des destinées humaines.

Robin Beuchat

Université de Genève

56. J. Rousset, La littérature de l'âge baroque en France, p. 8. 


\section{BIBLIOGRAPHIE}

\section{Textes}

Baudier, Michel, Inventaire de l'Histoire generalle des Turcz, Paris, H. le Gras et J. Guignar, 1631.

Busbecq, Ogier Ghislain de, Lettres du baron de Busbec, trad. par l'abbé de Foy, Paris, J.-B. Bauche et L. d'Houry, 1747.

Efendi, Tugi, La Mort du Sultan Osman, ou le retablissement de Mustapha sur le Throsne, traduit d'un manuscrit Turc, de la Bibliotheque du Roy, par Antoine Galland, Paris, Claude Barbin, 1678.

Furetière, Antoine, Dictionnaire universel, La Haye, A. et R. Leers, 1690.

Giustinian, Giorgio, Relazione (1627), in Relazioni di ambasciatori veneti al senato, vol. XIV, Costantinopoli, Relazioni inedite (15121789), a cura di M. P. Pedani-Fabris, Padova, Bottega d'Erasmo/ Aldo Ausilio Editore in Padova, 1996, p. 525-633.

Mercure François, t. 7, Paris, Jean Richer, 1622; t. 8, Paris, Jean Richer, 1623.

Montaigne, Michel de, Journal de voyage, éd. par Fausta Garavini, Paris, Gallimard, 1983.

NAUdÉ, Gabriel, Considérations politiques sur les coups d'Etat [1642], éd. par Frédérique Martin et Marie-Odile Perulli. Précédé de Pour une théorie baroque de l'action politique de Louis Marin, Paris, les Editions de Paris, 1989.

Pacifique de Provins, Lettre du Pere Pacifique de Provin [sic], predicateur Capucin, estant de present à Constantinople, envoyee au R.P. Joseph le Clerc, Predicateur du mesme Ordre, \& Deffiniteur de leur Province de Tours. Sur l'estrange mort du grand Turc, Empereur de Constantinople, Paris, François Huby, 1622.

-, Relation du voyage de Perse faict par le R.P. Pacifique de Provins, predicateur capucin, Paris, N. \& J. de la Coste, 1631. 
Purchas, Samuel B. D. (ed.), Hakluytus Posthumus or Purchas His Pilgrimes, Contayning a History of the World in Sea Voyages and Lande Travells by Englishmen and others, vol. VIII, Glasgow, James MacLehose and Sons, 1905 [1625].

Roe, Thomas, "The Death of Sultan Osman, and the setting up of Mustafa his Uncle, according to the Relation Presented to His Majestie», in Samuel Purchas, B. D. (ed.), Hakluytus Posthumus or Purchas His Pilgrimes, Contayning a History of the World in Sea Voyages and Lande Travells by Englishmen and others, vol. VIII, Glasgow, James MacLehose and Sons, 1905 [1625], p. 344-359.

-, The Negotiations of Sir Thomas Roe in his Embassy to the Ottoman Porte, from the Year 1621 to 1628 inclusive, London, Samuel Richardson, 1740.

SiRI, Vittorio, Il Mercurio overo historia de' correnti tempi, [s. l.], [s. n.], 1644.

Tristan L'Hermite, Osman [1656], éd. par Nicole Mallet, in CEuvres complètes, t. IV, éd. par Roger Guichemerre, Paris, Honoré Champion, 2001, p. 461-542.

\section{Travaux}

Bernardin, Napoléon-Maurice, Un précurseur de Racine: Tristan L'Hermite, sieur du Solier (1601-1655). Sa famille, sa vie, ses æuvres, Genève, Slatkine Reprints, 1967 (1895).

Giesey, Ralph E., Cérémonial et puissance souveraine. France, $X V^{\mathrm{e}}-X V I I^{\mathrm{e}}$ siècles, Paris, Armand Colin, 1987.

Hartog, François, Le miroir d'Hérodote. Essai sur la représentation de l'autre, Paris, Gallimard, 2001 (1980).

Kantorowicz, Ernst, The King's two Bodies, Princeton, Princeton University Press, 1957; trad. française, Les deux corps $d u$ roi, Paris, Gallimard, 1989.

Lancaster, Henry Carrington, A History of French Dramatic Literature in the Seventeenth Century, Baltimore, Johns Hopkins University Press, 1929-1942, vol. II.

Merlin-Kajman, Hélène, L'absolutisme dans les lettres et la théorie des deux corps. Passions et politique, Paris, Honoré Champion, 2000. 
Piterberg, Gabriel, An Ottoman Tragedy: History and Historiography at play, Berkeley/Los Angeles/London, University of California Press, 2003.

Rouillard, Clarence Dana, The Turk in French History Thought and Literature (1520-1660), Paris, Boivin, [1941].

Rousset, Jean, La littérature de l'âge baroque en France: Circé et le paon, Paris, Corti, 1985 [1954].

Vatin, Nicolas, Veinstein, Gilles, Le sérail ébranlé. Essai sur les morts, dépositions et avènements des sultans ottomans (XIVe-XIXe siècles), Paris, Fayard, 2003. 